\title{
Alterações hematológicas no paciente portador de HIV no momento do diagnóstico e seis meses após o início da terapia antiretroviral
}

\section{Hematologic changes in patients with HIV at the time of diagnosis and six months after starting antiretroviral therapy}

\author{
Nayro de Sousa Ferreira ${ }^{1,2}$. Kelsen Eulálio Dantas ${ }^{2,3}$. \\ 1 Universidade Federal do Ceará (UFC), Fortaleza, Ceará, Brasil. 2 Universidade Federal do Piauí (UFPI), Teresina, Piauí, Brasil. \\ 3 Fundação Municipal de Saúde, Instituto de Doenças Tropicais Natan Portela (IDTNP), Teresina, Piauí, Brasil.
}

\section{RESUMO}

A avaliação dos dados hematológicos associados à contagem de CD4+ e a quantificação da carga viral podem contribuir para o monitoramento da infecção pelo HIV. Objetivo: descrever as citopenias encontradas nos pacientes portadores de HIV no momento do diagnóstico e seis meses após o início da terapia antirretroviral. Metodologia: esta pesquisa é exploratória, descritiva e quantitativa, realizada entre janeiro e junho de 2018, através de questionário socioeconômico-demográfico e clínico. Resultados: a análise dos dados apontou resultados significativos em relação às alterações hematológicas dos pacientes com HIV/AIDS, no qual, após seis meses do diagnóstico, houve aumento dos níveis de Hemoglobina/Hematócrito $(\mathrm{P}=0,010 / 0,001)$, melhorando a anemia provocada pela doença, como também uma diminuição das taxas de Ferritina $(\mathrm{P}=<0,001)$, $\mathrm{LDH}(\mathrm{P}=0,006)$, Reticulócitos $(\mathrm{P}=0,001)$, TIBC $(\mathrm{P}=0,007)$ e T4 Livre $(\mathrm{P}=0,013)$, com efeito fisiológico benéfico para o organismo do paciente. Além disso, a carga viral atingiu níveis plasmáticos indetectáveis, elevando a quantidade de linfócitos CD4+, recuperando, assim, a imunidade. Conclusão: Todas as alterações hematológicas, isoladas ou combinadas, refletiram na ação direta do HIV sobre a medula óssea. Portanto, inflamações e/ou infecções oportunistas, deficiências nutricionais, efeito colateral de medicamentos e neoplasias estão correlacionadas diretamente com o grau de imunossupressão.

Palavras-chave: HIV. Hematologia. Agentes antirretrovirais.

\begin{abstract}
Evaluation of haematological data associated with CD4 + count and viral load quantification may contribute to the monitoring of HIV infection. Objective: To describe the cytopenias found in HIV patients at diagnosis and six months after initiation of antiretroviral therapy. Methodology: This is an exploratory, descriptive and quantitative research, conducted between January and June 2018, using a socioeconomic-demographic and clinical questionnaire. Results: Data analysis showed significant results regarding the hematological alterations of patients with HIV/AIDS, in which, after six months of diagnosis, there was an increase in hemoglobin/hematocrit levels $(\mathrm{P}=0.010 / 0.001)$, improving the anemia caused by the condition, as well as a decrease in Ferritin $(\mathrm{P}=<0.001)$, LDH $(\mathrm{P}=0.006)$, Reticulocyte $(\mathrm{P}=0.001)$, TIBC $(\mathrm{P}=0.007)$ and Free T4 $(\mathrm{P}=0.013)$ rates, beneficial physiological effect to the patient's organism. In addition, viral load reached undetectable plasma levels, increasing the amount of CD4 + lymphocytes, thus restoring immunity. Conclusion: All haematological changes, isolated or combined, reflected in the direct action of HIV on the bone marrow. Therefore, opportunistic inflammation and/or infections, nutritional deficiencies, medication side effects and neoplasms are directly correlated with the degree of immunosuppression.
\end{abstract}

Keywords: HIV. Hematology. Anti-retroviral agents.

Autor correspondente: Nayro de Sousa Ferreira, Rua Antilhon Ribeiro Soares, 5000, Santa Isabel, Teresina, Piauí. CEP: 64053-070. Telefone: +55 86 99976-8923. E-mail: drnayrosousa@gmail.com

Conflito de interesses: Não há qualquer conflito de interesses por parte de qualquer um dos autores.

Recebido em: 26 Ago 2019; Revisado em: 29 Jul 2020; Aceito em: 30 Jul 2020. 


\section{INTRODUÇÃO}

A pandemia da AIDS deu-se oficialmente em 1981, quando o Centers for Desease Control (CDC) publicou em seu boletim de 5 de junho o relato de cinco casos de homens jovens homossexuais com pneumonia por Pneumocistis carinii (hoje, Pneumocistis jirovecii), diagnosticados em Los Angeles, USA. A doença ocasionada por esse microorganismo era muito rara nessa época. Meses e anos após a descoberta da doença respiratória, surgiram centenas de casos de pneumonia ocasionadas pelo mesmo fungo, relatados pelo CDC. Observou-se então que a ocorrência da doença era diretamente ligada à imunossupressão que levava à inversão na relação de linfócitos, hoje conhecidos como $\mathrm{CD} 4+\mathrm{e} \mathrm{CD} 8+{ }^{1}$

Das alterações de órgãos e sistemas, as hematológicas são vistas desde a primeira descrição de infecção pelo HIV. As citopenias são observadas no momento do diagnóstico e têm relevância significativa, sendo a anemia com ocorrência de $70 \%$, linfopenia $70 \%$ e neutropenia $50 \%$. A gravidade das citopenias está diretamente relacionada com o grau de imunossupressão, sendo que entre 5 e 10\% dos pacientes já se mostram neutropênicos no início da infecção e isso pode predispor as doenças oportunistas em estágios avançados com variantes entre 50 a $70 \%$, de forma assintomática. ${ }^{2}$

A avaliação dos dados hematológicos associados à contagem de CD4+ e a quantificação da carga viral podem contribuir para o monitoramento da infecção pelo HIV e auxiliar na tomada de decisão a respeito da intervenção clínica mais adequada nesses pacientes, diminuindo assim a morbidade e mortalidade por complicações tratáveis no HIV. Na ausência de uma perspectiva de cura próxima com os meios presentes de tratamento, o início da terapia antiretroviral (TARV) é condicionada por considerações relativas ao estado clínico do paciente, à progressão do dano imunológico e à prevenção das comorbidades não infecciosas. ${ }^{1}$

No entanto, os benefícios associados ao uso da terapia antiretroviral são acompanhados por efeitos adversos, dentre eles: supressão da medula óssea e/ou anemia hemolítica evidenciada nesses pacientes. ${ }^{3}$

O objetivo geral desta pesquisa, foi descrever as citopenias encontradas nos pacientes portadores de HIV no momento do diagnóstico e seis meses após o início da terapia antirretroviral. Tendo como objetivos específicos: descrever o perfil socioeconômico-demográfico e clínico dos pacientes com HIV/AIDS; identificar as principais citopenias encontradas nos pacientes portadores de infecção pelo HIV; identificar as causas de citopenias nos pacientes portadores de infecção pelo HIV e descrever a evolução das citopenias após introdução da terapia antirretroviral.

\section{MATERIAL E MÉTODOS}

Tratou-se de um estudo descritivo de abordagem quantitativa que envolve o uso de técnicas padronizadas de coleta de dados e procura verificar e explicar sua influência sobre outras variáveis mediante a análise da frequência de incidências e de correlações estatísticas.

O estudo foi desenvolvido em um hospital estadual do município de Teresina-PI, localizado no centro da capital do Piauí, sendo referência no tratamento de doenças tropicais para as regiões Nordeste e Norte do Brasil, com atendimento ambulatorial e de urgência, possuindo cerca de 160 leitos distribuídos em 6 alas e uma Unidade de Terapia Intensiva.

A população foi composta por pacientes diagnosticados com HIV/AIDS quando foram internados no hospital ou em consulta ambulatorial com infectologista no período janeiro a junho de 2018, selecionados à medida que foram admitidos no serviço de saúde. Sendo excluídos todos os pacientes menores de dezoito anos e/ou que abandonaram o tratamento. Dessa forma, obteve-se uma amostra de 22 pacientes.

Foram incluídos no trabalho os pacientes com diagnóstico de HIV no momento da $1^{\text {a }}$ consulta com o infectologista ou que já tivessem o diagnóstico de infecção pelo HIV, mas sem acompanhamento com médico especialista e que nunca fizeram tratamento antiretroviral.

A coleta de dados se deu através da aplicação de um questionário socioeconômico-demográfico (Figura A) e outro questionário de avaliação hematológica (Figura B), onde foi escolhido local reservado, de acordo com as condições clínicas dos pacientes, a fim de manter a privacidade das informações.

Figura A. Questionário socioeconômico-demográfico e clínico.

\begin{tabular}{|c|c|c|}
\hline \multicolumn{3}{|c|}{$\begin{array}{c}\text { UNIVERSIDADE FEDERAL DO PIAUÍ } \\
\text { PROGRAMA DE RESIDÊNCIA MÉDICA EM INFECTOLOGIA }\end{array}$} \\
\hline \multicolumn{3}{|c|}{$\begin{array}{l}\text { PARÂMETROS LABORATORIA E CLÍNICOS DOS PACIENTES COM INFECÇÃO PELO } \\
\text { HIV OBTIDOS AO DIAGNÓSTICO E SEIS MESES APÓS INICIO DA TARV }\end{array}$} \\
\hline \multirow[b]{2}{*}{ Parâmetros } & \multicolumn{2}{|c|}{ Momento da coleta dos dados } \\
\hline & Data: ${ }^{\text {Ao diagnóstico }}$ & Data: 6 meses após \\
\hline \multicolumn{3}{|l|}{$\mathrm{Hb} / \mathrm{Hto}$} \\
\hline \multicolumn{3}{|l|}{ VCM / HCM } \\
\hline \multicolumn{3}{|l|}{ Leucócitos } \\
\hline \multicolumn{3}{|c|}{ Bastão/ Segmentados } \\
\hline \multicolumn{3}{|c|}{ Linfócitos/Monócitos } \\
\hline \multicolumn{3}{|c|}{ Eosinófilos/ Basósfilos } \\
\hline \multicolumn{3}{|l|}{ Plaquetas } \\
\hline \multicolumn{3}{|l|}{ Reticulócitos } \\
\hline \multicolumn{3}{|l|}{ LDH } \\
\hline \multicolumn{3}{|l|}{ Ferritina/ Ferro } \\
\hline \multicolumn{3}{|l|}{ TIBC } \\
\hline \multicolumn{3}{|c|}{ B12/ácido fólico } \\
\hline \multicolumn{3}{|c|}{ Uréia/ Creatinina } \\
\hline \multicolumn{3}{|l|}{ TSH/ T4I } \\
\hline \multicolumn{3}{|l|}{ Carga Viral } \\
\hline \multicolumn{3}{|c|}{ Contagem de CD4+ } \\
\hline \multicolumn{3}{|c|}{ IMC } \\
\hline \multicolumn{3}{|l|}{ Comorbidades } \\
\hline \multicolumn{3}{|c|}{ Medicamentos em uso } \\
\hline Intercorrências & & \\
\hline
\end{tabular}


Figura B. Questionário de avaliação hematológica.

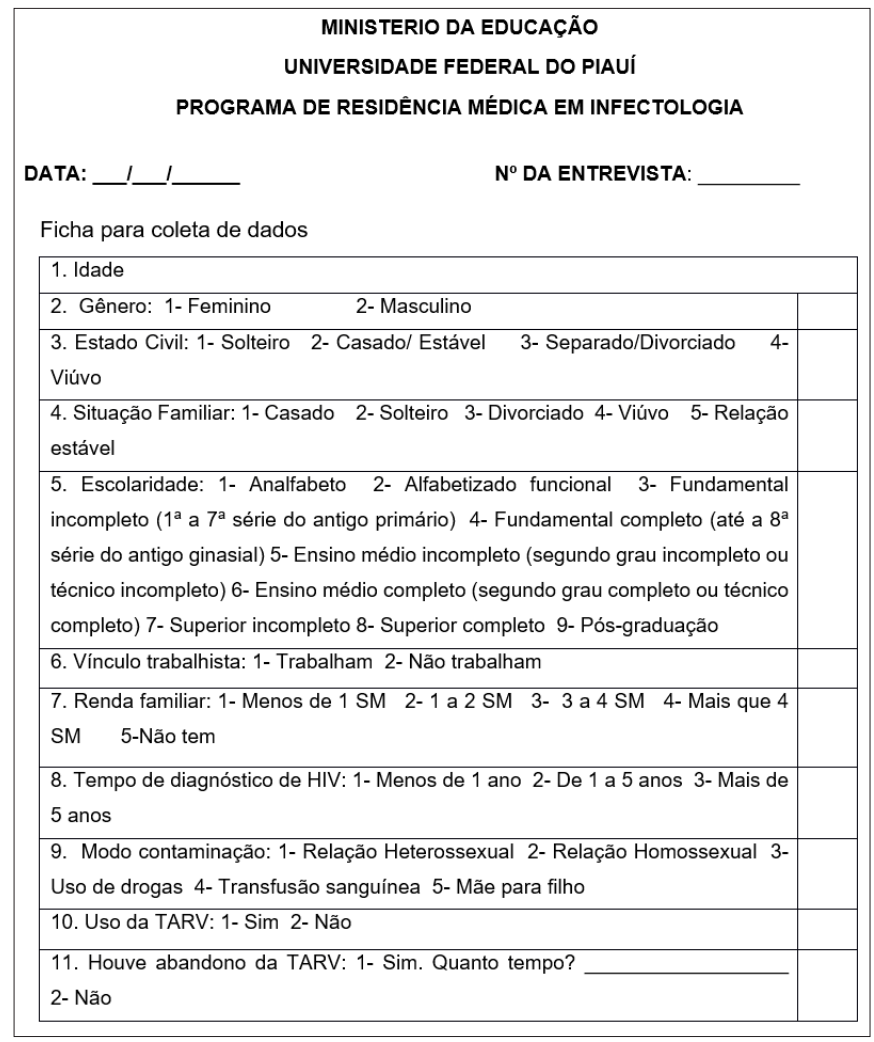

As variáveis de caracterização socioeconômica-demográfica foram: idade, gênero, estado civil, situação familiar, escolaridade, vínculo trabalhista e renda familiar. As relacionadas ao HIV foram: tempo de diagnóstico do HIV, modo de contaminação, uso da TARV.

Esse questionário foi aplicado em dois momentos: No dia da primeira consulta e/ou internação e após seis meses de tratamento com TARV. O questionário é composto por vários parâmetros hematológicos e clínicos do paciente. Vale ressaltar que os participantes na primeira consulta receberam uma numeração para sua codificação e foram orientados a trazêla seis meses após o tratamento com TARV. Esse número foi utilizado para manter o sigilo das informações e o anonimato dos pacientes no momento da análise dos dados.

Os dados obtidos foram armazenados em um banco eletrônico criado no programa Excel 2010 (Windows 7) e posteriormente analisados estatisticamente por meio do StatisticalPackage for the Social Sciences - SPSS (versão 20.0).

O projeto foi autorizado pela Comissão de Ética do hospital em estudo e ao Comitê de Ética em Pesquisa da Universidade Federal do Piauí (UFPI), atendendo aos aspectos éticos que regem as pesquisas envolvendo seres humanos, preconizados pela Resolução n 466/12 do Conselho Nacional de Saúde. ${ }^{4}$

\section{RESULTADOS}

Os resultados deste estudo relacionados ao perfil demográfico, indicaram uma prevalência de pacientes homens $(63,6 \%)$ com HIV/AIDS, de faixa etária entre 20 à 40 anos (68,2\%), apresentando como grau de escolaridade o ensino fundamental (45,5\%), com estado civil predominante solteiro (72,7\%), ativos no mercado de trabalho $(81,8 \%)$, porém com renda familiar menor que um salário mínimo, (54,6\%), como observado na Tabela 01 .

Quanto ao perfil clínico dos pacientes (Tabela 02) com HIV/ AIDS, todos tiveram confirmação do diagnóstico de HIV/ AIDS em menos de seis meses da coleta de dados e iniciaram tratamento com a Terapia Antiretroviral (TARV), no qual apenas um paciente $(4,5 \%)$ abandonou o tratamento durante o período de estudo. $\mathrm{O}$ modo de transmissão se apresentou equilibrado, onde $59,1 \%$ dos pacientes foram contaminados por HIV/AIDS por relação heterossexual e 40,9\% por relação homossexual.

As alterações hematológicas e demais variáveis clínicas (Tabela 03) demonstraram benefícios significativos com o uso da TARV: Houve aumento do IMC, melhora da anemia, diminuição da ferritina e do T4 livre, redução da carga viral para indetectável e elevação de CD4+, ou seja, desenvolve imunidade para a proteção de doenças oportunistas.

Tabela 1. Perfil sociodemográfico dos pacientes com HIV/AIDS participantes da pesquisa. Teresina-PI, 2018.

\begin{tabular}{|c|c|c|}
\hline Variáveis & $\mathbf{N}$ & $\%$ \\
\hline \multicolumn{3}{|l|}{ Sexo } \\
\hline Masculino & 14 & 63,6 \\
\hline Feminino & 08 & 36,4 \\
\hline \multicolumn{3}{|l|}{ Faixa etária } \\
\hline $20-40$ anos & 15 & 68,2 \\
\hline $41-80$ anos & 07 & 31,8 \\
\hline Média (D.P) & \multicolumn{2}{|c|}{$38,8(15,5)$} \\
\hline \multicolumn{3}{|l|}{ Escolaridade } \\
\hline Analfabeto & 04 & 18,2 \\
\hline Fundamental & 10 & 45,5 \\
\hline Médio & 03 & 13,6 \\
\hline Superior & 05 & 22,7 \\
\hline \multicolumn{3}{|l|}{ Estado civil } \\
\hline Solteiro (a) & 16 & 72,7 \\
\hline Casado(a)/União estável & 06 & 27,3 \\
\hline \multicolumn{3}{|l|}{ Vínculo trabalhista } \\
\hline Sim & 18 & 81,8 \\
\hline Não & 04 & 18,2 \\
\hline \multicolumn{3}{|l|}{ Renda Familiar } \\
\hline$<1 \mathrm{SM}$ & 12 & 54,6 \\
\hline $1,1-2,9 \mathrm{SM}$ & 05 & 27,7 \\
\hline $3,0-4,0 \mathrm{SM}$ & 05 & 27,7 \\
\hline Total & 22 & 100,0 \\
\hline
\end{tabular}

SM: Salário Mínimo (R\$=957,00); D.P: Desvio Padrão. 
Tabela 2. Perfil clínico dos pacientes com HIV/AIDS participantes da pesquisa. Teresina-PI, 2018.

\begin{tabular}{lcc}
\hline & N & \% \\
\hline Tempo diagnóstico do HIV & & \\
$\quad \leq 6$ meses & 22 & 100,0 \\
Modo de contaminação & & \\
$\quad$ Relação heterossexual & 13 & 59,1 \\
$\quad$ Relação homossexual & 09 & 40,9 \\
Uso de TARV & & \\
Sim & 22 & 100,0 \\
Não & 0 & 0,0 \\
Houve abandono da TARV & & \\
Sim & 01 & 4,5 \\
Não & 21 & 95,5 \\
\hline Total & $\mathbf{2 2}$ & $\mathbf{1 0 0 , 0}$ \\
\hline
\end{tabular}

TARV: terapia antirretroviral.

Tabela 3. Dados comparativos das variáveis clinicas dos pacientes com HIV/Aids participantes da pesquisa. Teresina-PI, 2018.

\begin{tabular}{|c|c|c|c|c|c|}
\hline \multirow{2}{*}{ Variáveis } & \multicolumn{2}{|r|}{ Antes } & \multicolumn{2}{|r|}{ Depois } & \multirow{2}{*}{$P^{*}$} \\
\hline & Média & IC95\% & Média & IC95\% & \\
\hline IMC (kg/m²) & 21,6 & $20,2-23,1$ & 23,4 & $22,0-24,9$ & 0,057 \\
\hline Hemoglobina (g/dL) & 10,9 & $8,3-13,6$ & 12,8 & $11,5-14,1$ & 0,010 \\
\hline Hematócrito (\%) & 28,6 & $24,4-32,9$ & 36,4 & $33,8-39,0$ & 0,001 \\
\hline VCM (fl) & 84,9 & $80,1-89,6$ & 88,8 & $86,7-90,8$ & 0,017 \\
\hline HCM (pg) & 27,2 & $25,6-28,9$ & 29,1 & $28,0-30,1$ & 0,036 \\
\hline Leucócitos (mm³) & 6587,0 & $4431,9-8742,2$ & 6020,0 & $5088,5-6951,6$ & 0,601 \\
\hline Eosinófilos (\%) & 0,7 & $0,4-1,0$ & 0,7 & $0,5-0,9$ & 1,000 \\
\hline Basófilos (\%) & 1,4 & $1,1-2,6$ & 0,8 & $0,6-1,3$ & 0,852 \\
\hline Plaquetas $(\mu \mathrm{l})$ & 192636,8 & $143710,0-241563,7$ & 191526,3 & $157143,9-225143,9$ & 1,000 \\
\hline Reticulócitos (\%) & 4,1 & $1,8-6,4$ & 0,9 & $0,7-1,2$ & $\mathbf{0 , 0 0 1}$ \\
\hline HDL (UI/L) & 600,6 & $239,5-961,7$ & 246,5 & $218,9-274,1$ & 0,006 \\
\hline Ferritina (ng/mL) & 1115,4 & $740,8-1490,0$ & 383,7 & $264,1-503,2$ & $<0,001$ \\
\hline Ferro (ng/mL) & 94,4 & $65,7-123,2$ & 75,8 & $55,9-95,7$ & 0,729 \\
\hline TIBC (ng/mL) & 294,6 & $244,6-344,6$ & 151,3 & $112,6-190,0$ & $\mathbf{0 , 0 0 7}$ \\
\hline B12 $(\mathrm{pg} / \mathrm{mL})$ & 401,0 & $323,2-478,9$ & 373,8 & $303,1-444,5$ & 0,198 \\
\hline Ácido fólico (ng/mL) & 10,4 & $9,0-11,7$ & 11,5 & $10,4-12,7$ & 0,587 \\
\hline Ureia (mg/dL) & 43,2 & $19,2-67,3$ & 27,0 & $25,4-28,4$ & 0,950 \\
\hline Creatina (ng/dL) & 1,2 & $0,6-1,7$ & 0,8 & $0,7-0,9$ & 0,068 \\
\hline TSH (ng/mL) & 2,1 & $1,4-2,8$ & 2,9 & $0,8-4,9$ & 0,929 \\
\hline T4 livre (ng/mL) & 7,1 & $5,8-8,4$ & 4,5 & $3,0-6,0$ & 0,013 \\
\hline Carga viral (cópias/mL) & 145379,8 & $79222,4-211537,1$ & & Indetectável & - \\
\hline CD4 + (células $\left./ \mathbf{m m}^{3}\right)$ & 119,3 & $77,2-161,3$ & 528,9 & $417,4-640,5$ & $<0,001$ \\
\hline
\end{tabular}

*Teste de Wilcoxon, IC95\%: Intervalo de confiança de 95\%, Dados em negrito significa diferença estatisticamente significa. 


\section{DISCUSSÃO}

$\mathrm{Na}$ análise dos dados socioeconômico-demográficos, 36,4\% $(n=8)$ são do sexo feminino e $63,6 \%(n=14)$ do sexo masculino. De acordo com o boletim epidemiológico de DST/AIDS 2014, a prevalência de HIV é maior entre os homens, sendo de 0,7 para indivíduos do sexo masculino e 0,4 para o sexo feminino, assim como os achados de Sousa-Gomes, ${ }^{5}$ em seu estudo que buscava avaliar os aspectos clínicos, epidemiológicos e laboratoriais da coinfecção HIV/LV, em que a população era predominantemente masculina.

Tradicionalmente, o homem é criado baseado em crenças e valores que reforçam sua masculinidade. Isso faz com que eles se considerem invulneráveis a doenças, que, dessa forma, é vista como sinal de fragilidade, o que acaba por contribuir para que cuidem menos de si e se tornem expostos às situações de risco com maior facilidade. ${ }^{6}$

A idade média dos entrevistados revelou uma população jovem de 20 a 40 anos, uma realidade refletida em todo o País, em que se observa, tradicionalmente, uma falta de cuidado com a própria saúde. Como podemos perceber, no estudo de Nuno Marques, ${ }^{7}$ sobre leishmaniose visceral e infecção por vírus da imunodeficiência humana, a média de idades foi de 38,2 anos com desvio padrão de $\pm 12,0$, mostrando que a concentração de casos de HIV no Brasil é predominante na faixa etária de 25 a 39 anos, em ambos os sexos. ${ }^{8}$

O grau de instrução dos pacientes estudados se apresentou da seguinte forma: $18,2 \%$ analfabetos, $45,5 \%$ possuíam o ensino fundamental, $13,6 \%$ o ensino médio e $22,7 \%$ tinham o ensino superior. Esta baixa escolaridade dos participantes encontrada neste estudo é similar à encontrada na literatura, pois pacientes soropositivos possuem apenas o ensino fundamental completo ou incompleto, sendo este fator que pode ser considerado um indicador para caracterizar o perfil socioeconômico de soropositivos, uma vez que a falta de estudo e acesso a informações indica uma condição de vulnerabilidade, ${ }^{9,10}$ como também influencia nas condições de vida, no estado de saúde, e no ingresso no mercado de trabalho. Observou-se nesta pesquisa que a renda familiar dos entrevistados $(54,6 \%)$ é menor que um salário mínimo. ${ }^{11,12}$

O estado civil do paciente com HIV neste estudo foi predominantemente solteiro, $72,7 \%(\mathrm{n}=16)$, porém, o modo de transmissão apresentou pequena diferença; 13 pacientes se contaminaram por meio heterossexual e 09 por meio homossexual. No mesmo raciocínio, Brasil (2014) ratifica que a forma de exposição entre homens é predominantemente por contato heterossexual, porém, enfatiza uma tendência de aumento na proporção entre homossexuais, como pode ser observado no estudo de Bertolini, ${ }^{13}$ em que houve predomínio de contato por relação homossexual. A forma de contágio por uso de drogas injetáveis vem sofrendo uma queda relevante nos últimos anos no Brasil.

No ponto de vista hematológico, as principais alterações no momento do diagnóstico foram a anemia, aumento da ferritina, do T4 livre e da carga viral, além da diminuição de CD4+ que, em alguns estudos, são apontadas como possíveis causas de letalidade no que diz respeito aos pacientes com HIV.

Como relatado na literatura, a anemia é comum em indivíduos infectados pelo HIV, ocorrendo em aproximadamente 30\% dos assintomáticos e em $60 \%$ a $70 \%$ daqueles com AIDS, e está relacionada com a qualidade de vida e sobrevida dos mesmos. ${ }^{14}$

Os doentes de SIDA com anemia tem uma taxa de mortalidade de 59\%. Esta taxa demonstrou ser muito alta quando comparada com a de mortalidade por outras causas, tais como a tuberculose (26\% de taxa de mortalidade), sepsis (22\%), HIVAN - doença de rins causada pelo VIH (12\%), sarcoma de Kaposi (10\%), cancro (7\%), demência (7\%) e outras doenças $(16 \%) \cdot{ }^{14,15}$

As causas de anemia nestes pacientes podem ser resultado da disfunção da medula óssea pelo vírus, que, durante a infecção, provoca alterações na secreção de citocinas, imunoglobulinas e proteínas de fase aguda, como resposta do sistema imunológico da célula hospedeira. ${ }^{16}$

As alterações hematológicas podem ser investigadas por meio de diferentes exames, contudo é ferramenta indispensável na confirmação da doença saber identificar tais alterações, que se reconhecidas e bem avaliadas possibilitam uma rápida terapêutica e redução da taxa de letalidade. ${ }^{17}$

Nos pacientes estudados, houve aumento da ferritina, proteína que possui a capacidade de agregar átomos de ferro, apartando-os para o compartimento de armazenamento do ferro, como também pode estar associada à circunferência abdominal e níveis de HDL-C elevados, hipertensão arterial, bem como a presença de resistência à insulina, ressaltando a forte inter-relação entre o metabolismo do ferro e a resistência à insulina. ${ }^{18}$

Todos os participantes deste estudo fazem o uso da TARV. É inegável que o uso da terapia, desde que foi introduzido no Brasil, em 1996, vem reduzindo significativamente a morbimortalidade por AIDS e aumentando a sobrevida dos infectados. Além do impacto clinico oportuno, o uso da TARV é importante na redução da transmissão do HIV. No entanto, é necessário considerar o grau de adesão ao tratamento como estratégia para o sucesso da terapia. ${ }^{6}$

Em relação ao uso da TARV seis meses após o diagnóstico, esta pesquisa observou benefício significativo em todos os aspectos clínicos e hematológicos, não apenas no declínio da carga viral e no aumento da contagem de TCD4+, mas no aumento do IMC, melhora da anemia e da dislipidemia, diminuição da ferritina e do T4 livre.

Durval relataram que o uso da TARV induz uma importante e sustentada supressão na replicação do vírus, o que ocasiona a diminuição da carga viral. Portanto, menores cargas virais são responsáveis por maiores níveis de TCD4+ nos pacientes tratados, sendo referidas como importantes instrumentos 
de monitorização laboratorial de falha virológica, a ser investigada no manejo e acompanhamento da terapia com antirretrovirais. ${ }^{3,19}$

\section{CONCLUSÃO}

A anemia nos pacientes soropositivos tem características hematológicas de anemia das doenças crônicas, no qual é observado que com o uso do TARV se tem um aumento do CD4+ e queda da carga viral, onde isto influenciará no aumento do IMC, na redução da resposta inflamatória crônica,

\section{REFERÊNCIAS}

1. Alencar TM, Nemes MI, Velloso MA. Transformações da "aids aguda" para a "aids crônica": percepção corporal e intervenções cirúrgicas entre pessoas vivendo com HIV e aids. Ciência Saúde Coletiva. 2008;13(6):1841-9.

2. Friel TJ, Scadden DT. Hematologic manifestations of HIV infection: thrombocytopenia and coagulation abnormalities. 2014 [acesso em: 8 jan 2016]. Disponível em: https://somepomed.org/ articulos/contents/mobipreview.htm?21/23/21873?source=related link.

3. Duval X, Journot V, Leport C, Chêne G, Dupon M, Cuzin L, et al. Incidence of and risk factors for adverse drug reactions in a prospective cohort of HIV-infected adults initiating protease inhibitor-containing therapy. Clin Infect Dis. 2004;39(2):24855 .

4. Brasil, Ministério da saúde. Boletim epidemiológico HIV/AIDS. Brasília: Ministério da Saúde; 2014 [acesso em: 28 set 2018]. Disponível em: http://www.aids.gov.br/pt-br/node/73.

5. Sousa-Gomes ML, Maia-Elkhoury AN, Pelissari DM, Lima FE Junior, Sena JM, Cechinel MP. Coinfecção Leishmania-HIV no Brasil: aspectos epidemiológicos, clínicos e laboratoriais. Epidemiol Serv Saúde. 2011;20(4):519-26.

6. Brasil. Ministério da saúde. Secretaria de vigilância em saúde. Recomendações para terapia antirretroviral em adultos infectados pelo HIV. Brasília: Ministério da Saúde; 2008 [acesso em: 3 out 2018]. Disponível em: http://www.aids.gov.br/pt-br/pub/2007/ recomendacoes-para-terapia-antirretroviral-em-adultos-infectadospelo-hiv-2008

7. Marques N, Cabral S, Sá R, Coelho F, Oliveira J, Saraiva da Cunha JG, et al. Leishmaniose visceral e infecção por vírus da imunodeficiência humana na era da terapêutica anti-retrovírica de alta eficácia. Acta Med Port. 2007;20:291-8.

8. Brasil. Ministério da Saúde. Secretaria de Atenção à Saúde. Departamento de Atenção Básica. HIV/Aids, hepatites e outras DST. Brasília: Ministério da Saúde; 2006 [acesso em: 5 set 2018]. (Cadernos de Atenção Básica, n. ${ }^{\circ}$ 18. Série A, Normas e manuais técnicos). Disponível em: http://bvsms.saude.gov.br/bvs/publicacoes/ abcad18.pdf

9. Alves LA, Silveira MF, Pinheiro CA, Stoffel PC, Pieniz C, Rozenthal RM. Prevalência de alterações hematológicas em mulheres com HIV/Aids assistidas em serviço especializado: relato de série de casos. Revista da AMRIGS. 2011;55(4):324-6. e consequentemente, da anemia observada no momento do diagnóstico. Portanto, os dados que esta investigação se ampara - a efetividade de melhora clínica e laboratorial com o uso da TARV e do diagnóstico precoce- é legítima e complexa, necessitando de mais estudos desta natureza, para que se consiga identificar as reais causas das alterações aqui descritas, com amostras significativas em que seja possível relacionar as citopenias aos achados clínicos, visto a responsividade satisfatória precisa ser amparada e incentivada para o desenvolvimento de estudos qualitativos que avancem neste campo de investigação.

10. Brasil. Ministério da saúde. Secretaria de vigilância em saúde. Protocolo clínico e diretrizes terapêuticas para manejo da infecção pelo HIV em adultos. Brasília: Ministério da Saúde; 2013 [acesso em: 21 out 2018]. Disponível em: http://www.aids.gov.br/pt-br/ pub/2013/protocolo-clinico-e-diretrizes-terapeuticas-para-manejoda-infeccao-pelo-hiv-em-adultos

11. Cruz FS. Famílias vivendo com HIV/AIDS em Campo Grande: estratégias de enfrentamento, apoio social e qualidade de vida [dissertação]. Campo Grande: Universidade Católica Dom Bosco; 2008 [acesso em: 12 ago 2018]. Disponível em: https://site.ucdb. br/public/md-dissertacoes/7748-familias-vivendo-com-hiv-aidsem-campo-grande-estrategias-de-enfrentamento-apoio-social-equalidade-de-vida.pdf

12. Santos EM, França I Junior, Lopes F. Qualidade de vida de pessoas vivendo com HIV/AIDS: enfrentamento, suporte social e qualidade de vida. Psicol Reflex Crit. 2007;2(18):188-95.

13. Bertoni RF, Bunn K, Silva J, Traebert J. Perfil demográfico Socioeconômico dos portadores de HIV/AIDS do ambulatório de controle de DST/AIDS de São Jose, SC. Arq Catarin Med. 2010 [acesso em: 28 set 2018];39(4):75-9. Disponível em: http://www. acm.org.br/acm/revista/pdf/artigos/835.pdf

14. Oliveira OC, Oliveira RA, Souza LR. Impacto do tratamento antirretroviral na ocorrência de macrocitose em pacientes com HIV/ AIDS do município de Maringá, Estado do Paraná. Rev Soc Bras Med Trop. 2011 [acesso em: 19 maio 2018];44(1):35-9. Disponível em: $\quad$ http://www.scielo.br/scielo.php?script=sci_arttext\&pid $=$ S0037-86822011000100009

15. Hayden E. Anaemia is a risk factor for mortality in patients with AIDS. 2009 [acesso em: 9 jan 2016]. Disponível em: https://www. aidsmap.com/news/apr-2009/anaemia-risk-factor-mortality-patientsaids

16. Claster S. Biology of anemia, differential diagnosis, and treatment options in human immunodeficiency virus infection. J Infect Dis. 2002;185(Supl 2):S105-9.

17. Moreira EA. Aspectos hematológicos de pacientes com Leishmaniose Visceral. São José do Rio Preto: Academia de Ciência e Tecnologia; 2012 [acesso em: 13 mar 2019]. Disponível em: http:// www.ciencianews.com.br/arquivos/ACET/IMAGENS/revista virtual/hematologia/hemato21.pdf

18. Monteiro JP, Cunha DF, Cunha SF, Santos VM, Silva-Vergara ML, Correia D, et al. Resposta de fase aguda, subnutrição e estado 
nutricional do ferro em adultos com AIDS. Rev Soc Bras Med Trop. 2000 [acesso em: 8 março 2019];33(2):175-80. Disponível em: http://www.scielo.br/scielo.php?script=sci_arttext\&pid=S0037$86822000000200003 \& \operatorname{lng}=\mathrm{en} \& \mathrm{nrm}=$ iso
19. Dhurve SA, Dhurve AS. Bone Marrow Abnormalities in HIV Disease. Mediterr J Hematol Infect Dis. 2013 [acesso em: 8 mar 2019];5(1):33. Disponível em: https://www.ncbi.nlm.nih.gov/pmc/ articles/PMC3684351/

\section{Como citar:}

Ferreira NS, Dantas KE. Alterações hematológicas no paciente portador de HIV no momento do diagnóstico e seis meses após o início da terapia antiretroviral. Rev Med UFC. 2021;61(1):1-7. 